\title{
Two Clade A Phosphatase 2Cs Expressed in Guard Cells Physically Interact With Abscisic Acid Signaling Components to Induce Stomatal Closure in Rice
}

\author{
Myung Ki Min ${ }^{1 \dagger}$, Eun-Hye Choi ${ }^{1 \dagger}$, Jin-Ae Kim ${ }^{1}$ In Sun Yoon ${ }^{2}$, Seungsu Han ${ }^{3}$, Yeongmok Lee ${ }^{3}$, Sangho Lee ${ }^{3}$ and \\ Beom-Gi Kim ${ }^{1 *}$ (D)
}

\begin{abstract}
Background: The core ABA signaling components functioning in stomatal closure/opening, namely ABA receptors, phosphatases, SnRK2s and SLAC1, are well characterized in Arabidopsis, but their functions in guard cells of rice have not been extensively studied.

Results: In this study, we confirmed that OSSLAC1, the rice homolog of AtSLAC1, is specifically expressed in rice guard cells. Among the rice SAPKS, SAPK10 was specifically expressed in guard cells. In addition, SAPK10 phosphorylated OSSLAC1 in vitro and transgenic rice overexpressing SAPK10 or OsSLAC1 showed significantly less water loss than control. Thus, those might be major positive signaling components to close stomata in rice. We identified that only OsPP2C50 and OsPP2C53 among 9 OsPP2CAs might be related with stomatal closure/ opening signaling based on guard cell specific expression and subcellular localization. Transgenic rice overexpressing OsPP2C50 and OsPP2C53 showed significantly higher water loss than control. We also characterized the interaction networks between OsPP2C50 and OsPP2C53, SAPK10 and OsSLAC1 and found two interaction pathways among those signaling components: a hierarchical interaction pathway that consisted of OsPP2C50 and OsPP2C53, SAPK10 and OsSLAC1; and a branched interaction pathway wherein OsPP2C50 and OSPP2C53 interacted directly with OsSLAC1.

Conclusion: $\mathrm{OsPP2C50}$ and $\mathrm{OsPP2C} 53$ is major negative regulators of $\mathrm{ABA}$ signaling regarding stomata closing in rice. Those can regulate the OsSLAC1 directly or indirectly thorough SAPK10.
\end{abstract}

Keywords: Rice, Guard cell, ABA, Stomata, Phosphatase

\section{Background}

In plants, stomatal apertures represent the main gateways for exchange of gases, such as $\mathrm{O}_{2}, \mathrm{CO}_{2}$, and water vapor between the leaf and the atmosphere. Stomata open or close in response to light, $\mathrm{CO}_{2}$ concentration, atmospheric humidity, ozone, and small stress-generated signaling molecules such as abscisic acid (ABA), jasmonic acid, $\mathrm{Ca}^{2}$ ${ }^{+}$, nitric oxide, and reactive oxygen species (Bauer et al. 2013; Hetherington and Woodward 2003; Kim et al. 2010;

\footnotetext{
* Correspondence: bgkimpeace@gmail.com

${ }^{\dagger}$ Myung Ki Min and Eun-Hye Choi contributed equally to this work.

${ }^{1}$ Metabolic Engineering Division, Department of Agricultural Biotechnology, National Institute of Agricultural Sciences, Rural Development Administration, Jeonju 54875, Republic of Korea

Full list of author information is available at the end of the article
}

Schroeder et al. 2001). The stomatal aperture is a pore surrounded by two guard cells, which can be expanded or shrunk by changes in turgor pressure. Channels such as the $\mathrm{K}^{+}$-outward-rectifying channel (GORK), $\mathrm{K}^{+}$-inward-rectifying channel (KAT1), rapid-type anion channel and slow-type anion channel (SLAC1) regulate the turgor pressure of guard cells by transporting ions including $\mathrm{K}^{+}$, $\mathrm{Cl}^{-}$, and malate (Ache et al. 2000; Anderson et al. 1992; Kim et al. 2015; Schachtman et al. 1992; Vahisalu et al. 2008). Among these channels, SLAC1 has been reported to be crucial for closing the stomata in Arabidopsis thaliana (Arabidopsis). Activated SLAC1 depolarizes the plasma membrane to activate GORK and push $\mathrm{K}^{+}$out 
from the guard cell, thereby reducing the turgor pressure and closing the stomatal aperture (Sirichandra et al. 2009).

ABA and $\mathrm{Ca}^{2+}$ signaling regulate SLAC1 activity via phosphorylation and dephosphorylation. Serine 120 (Ser120) of Arabidopsis SLAC1 is phosphorylated and activated in the presence of ABA, mainly by OPEN STOMATA 1 (OST1; also called SnRK2E or SnRK2.6), a SNF1-RELATED PROTEIN KINASE2 (SnRK2) family protein (Geiger et al. 2009; Lee et al. 2009; Vahisalu et al. 2010). In addition, CALCIUM-DEPENDENT PROTEIN KINASE 6/21/23 (CPK 6/21/23) and CBL-INTERACTING PROTEIN KINASEs (CIPKs), which play roles in $\mathrm{Ca}^{2}$ ${ }^{+}$-dependent signaling, activate SLAC1 by phosphorylating Ser59 in response to $\mathrm{Ca}^{2+}$ signaling (Brandt et al. 2012; Geiger et al. 2010; Maierhofer et al. 2014). The clade A type $2 \mathrm{C}$ protein phosphatases (PP2CAs) play negative regulatory roles in stomatal closure via $\mathrm{ABA}$ and $\mathrm{Ca}^{2+}$ signaling (Mustilli et al. 2002; Zhang et al. 2014). PP2CAs suppress ABA signaling by interacting with and inactivating OST1 and CPK6. On other hand, PP2CAs directly interact with and inactivate SLAC1 by dephosphorylation, counteracting its phosphorylation by OST1 or CPKs in response to ABA or $\mathrm{Ca}^{2+}$ signaling (Brandt et al. 2012; Geiger et al. 2009; Lee et al. 2009).

The core ABA signaling components, which consist of ABA receptors, PP2CAs and SnRK2s, are well conserved among plants, even though monocots and dicots have different types of guard cells, dumbbell and kidney types, respectively (Hauser et al. 2011; Schafer et al. 2018). Thus, it is necessary to systematically identify and compare the differences and similarities among ABA signaling components functioning in monocot and dicot guard cells. To date, there are few reports regarding core ABA signaling components focused on monocot guard cells (Schafer et al. 2018; Sun et al. 2016). Recently it has been reported that monocot SLAC1s such as OsSLAC1 and HvSLAC1 of rice and barley require extracellular nitrate for activation but dicot SLAC1s do not (Schafer et al. 2018; Sun et al. 2016). However, it was not known whether the molecular mechanisms of ABA signaling are different between Arabidopsis and rice (Oryza sativa).

Here, we identified the core ABA signaling components functioning in rice guard cells and characterized their interaction networks. This study provides useful information about the molecular mechanisms of monocot guard cell movement. Furthermore, this information will contribute to improved water usage efficiency in crops through guard cell regulation.

\section{Results}

OsSLAC1 is Expressed in Guard Cell and Overexpression Lines of OSSALC1 Lose Less Water in Rice

The guard cell-expressed AtSLAC1 functions as an S-type anion channel and is one of the major targets of $\mathrm{ABA}$ signaling for stomatal closure in Arabidopsis. Previously, OsSLAC1 (Os04g18530) was identified as the most closely related rice protein to AtSLAC1 (Additional file 1: Figures S1A) (Kusumi et al. 2012). OsSLAC1 has been reported to regulate $\mathrm{CO}_{2}$ homeostasis in rice and function as a nitrate-selective anion channel when expressed in Xenopus oocytes (Kusumi et al. 2012; Sun et al. 2016), and to present in rice guard cells confirmed by own promoter GUS expression analysis (Kusumi et al. 2017). We also analyzed gene expression profiles of the rice S-type anion channels in different tissues using the Rice Expression Profile Database (http://ricexpro.dna. affrc.go.jp) and Genevestigator (http://genevestigator. com) (Additional file 1: Figure S1B and Additional file 6: Figure S6A). Among the S-type anion channels of rice, OSSLAC1 was expressed predominantly in the leaf blade, even though other homologues are expressed in roots or stems. When we examined the expression of OsSLAC1 using RT-qPCR in two-week-old seedlings, OsSLAC1 expression was dramatically higher in shoots than in roots (Fig. 1a). When total RNA was isolated from leaf blade, leaf sheath, stem and flower tissues of mature rice (80-day-old plants), OsSLAC1 expression was found to be higher in leaf tissues (including leaf blade and sheath) than in other tissues (Fig. 1b). To examine the tissue-specific expression of OSSLAC1 in more detail, we constructed transgenic rice lines (D03) harboring an OSSLAC1 promoter- $\beta$-glucuronidase (GUS) reporter vector (pOsSLAC1::GUS). Leaves showed strong X-Gluc staining, but roots were not stained in 10-day-old transgenic rice seedlings (Fig. 1c). Mature transgenic rice, including pOsSLAC1-GUS, did not express GUS in culm, tiller or flower. The epidermis of stained seedling leaves was observed under a microscope and showed GUS staining specifically in guard cells (Fig. 1c). We sectioned stained seedling leaves to dissect the expression in detail and we confirmed that X-Gluc staining was detected only in the guard cells and not in mesophyll cells or other epidermal cells (Fig. 1d). Thus, we can conclude that OsSLAC1 is expressed specifically in guard cell. We also confirmed the subcellular localization of OsSLAC1-GFP. The fluorescence signal of OsSLAC1-GFP overlapped with the signal of a plasma membrane-localized marker (PM-mCherry) in rice protoplasts (Fig. 1e).

To identify the effects of OsSLAC1 in stomatal closure, we constructed transgenic rice lines overexpressing OsSLAC1 (B76). Three OsSLAC1 over-expression lines were selected based on gene expression level (Additional file 4: Figure S4A). Transgenic plant lines harboring the empty vector were used as the control and named as A8. Water loss ratios of the OSSLAC1-overexpression lines (B76) were significantly less than those of the control (A8) from 1 to $5 \mathrm{~h}$ in all three lines (Fig. 1f). 


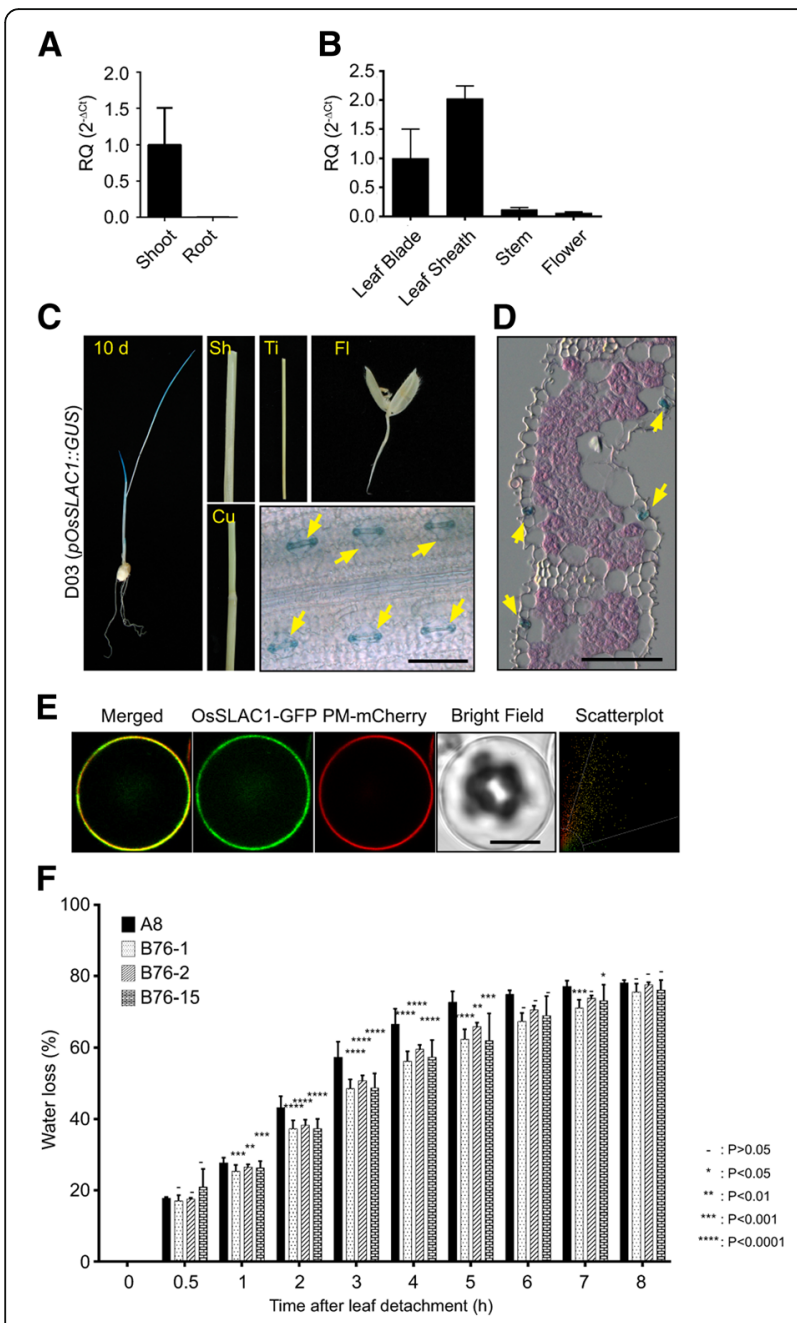

Fig. 1 OSSLAC1 is specifically expressed in rice guard cells and OsSLAC1-GFP localizes to the plasma membrane in rice protoplasts. a RT-qPCR analysis of OsSLAC1 gene expression in two-week-old seedlings. The generated $R Q$ (relative quantity, $2^{-\Delta C t}$ ) was compared to the value of expression on shoot. OsUbi5 was used as endogenous control. The values depict the average and \pm SD with 3 replications. b RT-qPCR analysis of OsSLAC1 gene expression in different tissues of mature rice ( 80 days old). The values were compared to the expression level on leaf blade. c Histochemical $\beta$-glucuronidase analysis of OSSLAC1 promoter-GUS transgenic rice. Left image is a 10-day-old seedling. Sh (sheath), Ti (tiller), FI (flower), Cu (culm) and leaf blade epidermis (bottom right). Scale bars are $50 \mu \mathrm{m}$. d A section of paraffin-embedded leaf blade stained with X-Gluc (blue) and eocin. Yellow arrows indicate guard cells. Scale bars are $50 \mu \mathrm{m}$. e Subcellular localization of OSSLAC1-GFP and marker protein in rice protoplast. PM-mCherry is a plasma membrane localization marker. The scatterplot shows the values of fluorescence of the pixels across the two channels. Scale bars are $10 \mu \mathrm{m}$. f Water loss assays of OsSALC1-overexpressing transgenic lines (B76-1, 2 and 15) and control line (A8: empty vector transgenic plant). The values depict the average and \pm SD with 3 replications. ANOVA analysis was performed by comparing with water loss of A8 plant

\section{SAPK10 is Expressed in Rice Guard Cells and SAPK10} Overexpression Lines Present Lower Water Loss in Detached Leaves

OST1 (SnRK2-6) is a crucial positive regulator of ABA signal transduction in Arabidopsis guard cells (Brandt et al. 2012; Geiger et al. 2009; Mustilli et al. 2002). Rice orthologous of Arabidopsis SnRK2s are called Osmotic Stress/ABA-Activated Protein Kinases (SAPKs) and ten $S A P K$ s are present in the rice genome (Kobayashi et al. 2004). We analyzed previously published rice cell-specific microarray data (GEO accession: Go Series GSE13161) to determine which of the ten SAPKs are expressed in rice guard cells (Jiao et al. 2009). We found that, of the ten SAPKs, SAPK10 was most prominently expressed in rice guard cells (Fig. 2a). Thus, we experimentally confirmed the expression of $S A P K 10$ in different tissues using RT-qPCR. SAPK10 expression was predominant in shoots compared to roots of young rice seedlings (two weeks old) (Fig. 2b). SAPK10 expression levels were higher in leaf tissues (including leaf blade and sheath) than in other tissues of mature rice ( 80 days old) (Fig. 2c). Results were similar to microarray data base search at Genevestigator (Additional file 6: Figure S6C). To experimentally validate the guard cell-specific expression of SAPK10, we generated transgenic rice lines expressing GUS driven by the SAPK10 promoter ( $p O s S A P K 10:: G U S$, named D06). The $S A P K 10$ promoter drove expression of GUS in the seeds and shoots of young seedlings and in the leaf blade and node of mature plants predominantly, even though lower levels of expression were also observed in the sheath and culm of the mature plant (80 days old) (Fig. 2d). However, we could not observe the expression of SAPK10 in flower and tiller. Microscopic observation of the leaf epidermis and leaf blade sections showed that X-Gluc staining was detected in guard cells predominantly (Fig. 2e). We generated transgenic rice lines overexpressing SAPK10 and three SAPK10 over-expression lines named C7 were selected for water loss assays. The expression levels of SAPK10 were confirmed by RT-qPCR analysis (Additional file 4: Figure S4B). All three SAPK10-overexpression lines lost significantly less water than the control (A8) (Fig. 2f).

\section{SAPK10 Interacts With and Phosphorylates OsSLAC1}

When OST1 is activated by ABA signaling, it interacts with and phosphorylates AtSLAC1, thereby activating AtSLAC1 and inducing stomatal closure. Thus, we examined whether SAPK10 can interact with and phosphorylate OsSLAC1. First, we performed yeast two-hybridization assays. Since OsSLAC1 is a plasma membrane protein, the $\mathrm{N}$-terminal and $\mathrm{C}$-terminal regions (N-OsSLAC1 and C-OsSLAC1) where OsSLAC1 protrudes into cytosol were cloned and used as prey. Only N-OsSLAC1 demonstrated a weak interaction with SAPK10 (Fig. 3a). 


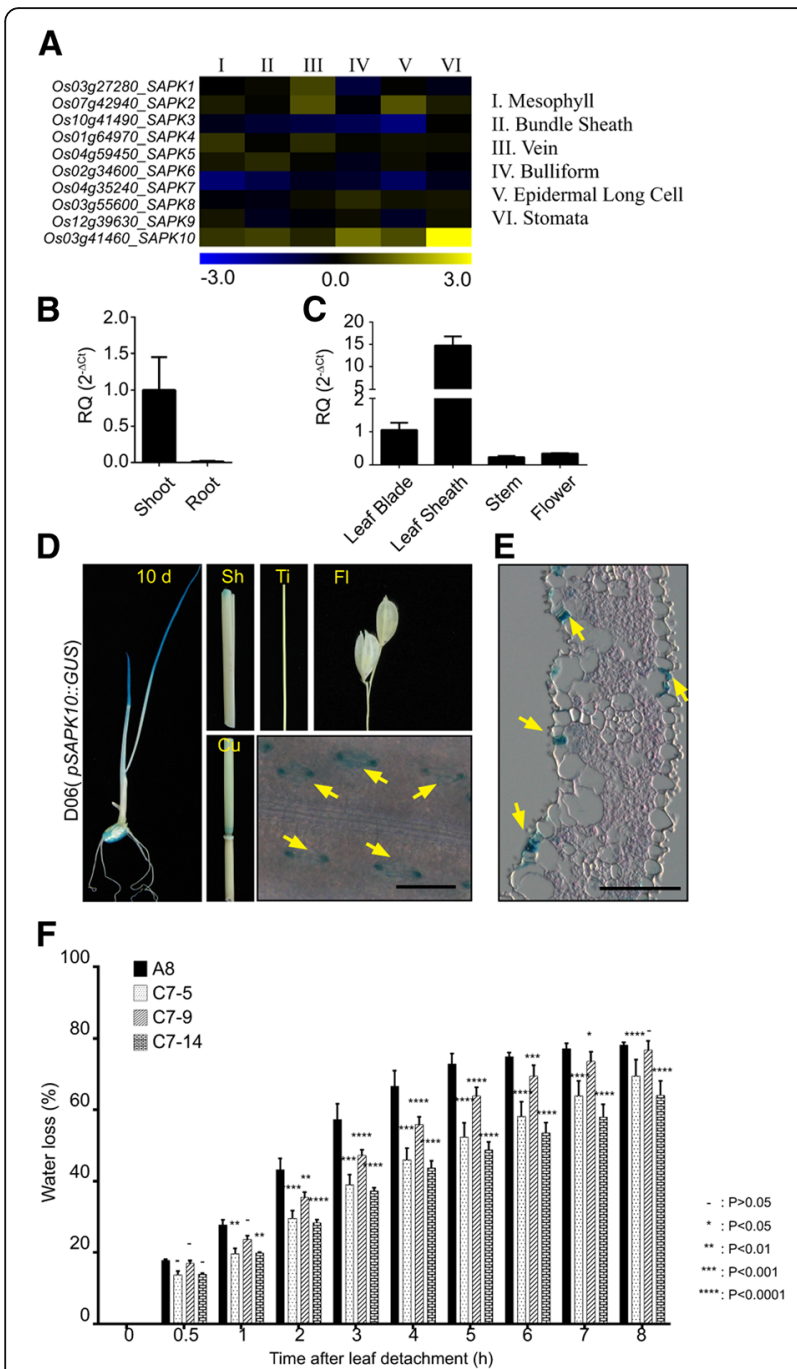

Fig. 2 SAPK10 is expressed dominantly in rice guard cells and OsSAPK10-overexpressing transgenic rice presents less water loss. a Gene expression profiles of ten SAPKs in different rice cell types were presented as a heat map by using transcriptome analysis data from rice cells, black to yellow displayed increasing, black to blue displayed decreasing. $\mathbf{b}$ RT-qPCR analysis of OsSLAC1 gene expression in two-week-old seedlings. The generated RQ (relative quantity, $2^{-\Delta c t}$ ) was compared to the value of expression on shoot. OsUbiqutin5 was used as endogenous control. The values depict the average and \pm SD with 3 replications. c RT-pPCR analysis of OsSLAC1 gene expression in different tissues of mature rice ( 80 days old). The values were compared to the expression level on leaf blade. d Histochemical $\beta$-glucuronidase analysis of SAPK10 promoter-GUS transgenic rice. Left image is a 10-day-old seedling. Sh (sheath), Ti (tiller), Fl (flower), $\mathrm{Cu}$ (culm) and leaf blade epidermis. Scale bars are $50 \mu \mathrm{m}$. e A section of paraffin-embedded leaf blade stained with $X-G l u c$ and eocin. Yellow arrows indicate guard cells. Scale bars are $50 \mu \mathrm{m}$. $\mathbf{f}$ Water loss assays of SAPK10-overexpressing transgenic lines (C7-5, 9 and 14) and control line (A8: empty vector transgenic rice). The picks depict the average and \pm SD with 3 replications. ANOVA analysis was performed by comparing with water loss of A8 plant

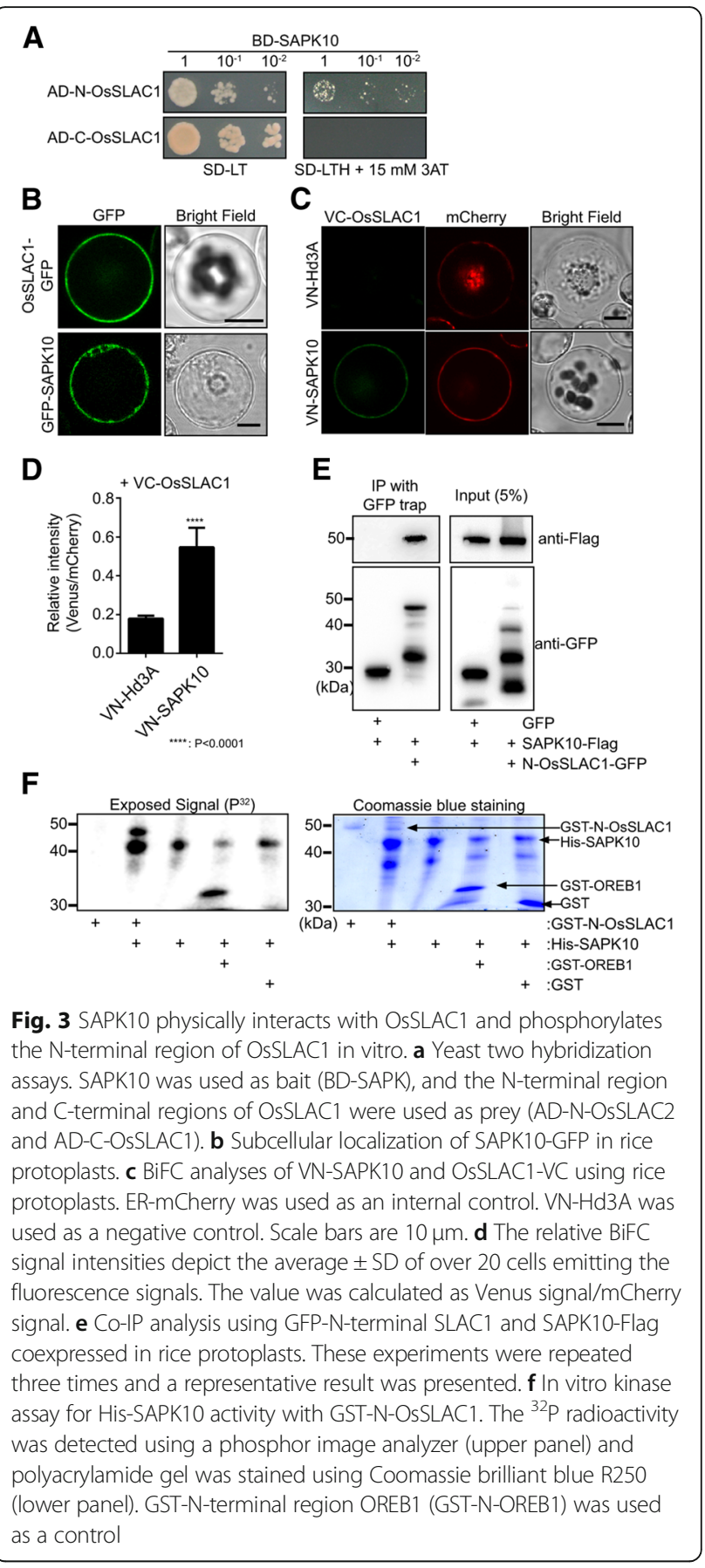

We further investigated the interaction and subcellular localization of this complex using a bimolecular fluorescence complementation (BiFC) assay. A yellow fluorescence signal was observed on the plasma membrane when VC-OsSLAC1 and VN-SAPK10 were co-expressed in rice protoplasts but not in the negative control (VN-Hd3A and VC-OsSLAC1). The fluorescence values of VN-SAPK10 and VC-SLAC1 were three times higher than VN-Hd3A and VC-SLAC1 (Fig. 3c and 
d). We also characterized the subcellular localization of GFP-SAPK10, which was detected in the cytosol of rice protoplasts (Fig. 3b). Co-immunoprecipitation (co-IP) analysis also confirmed the physical interaction between N-OsSLAC1-GFP and SAPK10-Flag in rice protoplast (Fig. 3e).

To explore whether SAPK10 is able to phosphorylate OsSLAC1, we expressed glutathione S-transferase (GST)-tagged N-OsSLAC1 (GST-N-OsSLAC1) and 6XHis-tagged SAPK10 (His-SAPK10) in E. coli and used the purified proteins to carry out an in vitro phosphorylation assay. His-SAPK10 phosphorylated itself, GST-N-OsSLAC1 and the N-terminal region of OREB1 (GST-N-OREB1), used as positive control (Fig. 3f) (Chae et al. 2007). Based on these results, we conclude that SAPK10 is a functional orthologue of OST1, which physically interacts with OsSLAC1 and can phosphorylate its $\mathrm{N}$-terminal region.

\section{OsPP2C50 and OsPP2C53 are Localized in the Cytosol and are Expressed Predominantly in Guard Cells}

Clade A phosphatase 2Cs (PP2CAs) are negative regulators of SAPK/SnRK2 in ABA signaling, in both Arabidopsis and rice, and we identified nine OsPP2CAs in the rice genome (Kim et al. 2012). To identify which OsPP2CAs among 9 OsPP2CAs function in guard cell to regulate SAPK10 and OsSLAC1, we surveyed the subcellular localization of OsPP2CAs and the expression of the $O s P P 2 C A \mathrm{~s}$ in guard cells. First, we characterized the subcellular localization of all nine OsPP2CAs in rice protoplasts. The OsPP2CAs were clearly divided into two groups based on subcellular localization: one with nucleus-specific localization, and the other with non-nucleus-specific localization, resembling cytosolic, ER-like or Plasma membrane localization and distributed ubiquitously in cell (Fig. 4a). These two groups corresponded to the two clades observed in phylogenetic analysis which are presented in the right side of subcellular localization (Fig. 4a). As candidates to regulate SAPK10, we chose the three OsPP2CAs (OsPP2C06 (Os01g40094), OsPP2C53 (Os05g51510) and OsPP2C50 (Os05g46040)) not located specifically in the nucleus, and then explored whether they were expressed in rice guard cells. We generated transgenic rice lines named D04, E48, E46 harboring $p O s P$ P2C06::GUS, pOsPP2C53::GUS and pOsPP2C50::GUS reporter vectors, respectively. We found that the promoters of OsPP2C50 and OsPP2C53 drove expression of GUS in guard cells, but that of OsPP2CO6 did not, regardless of $\mathrm{ABA}$ treatment (Fig. 4b, c, d). The promoter of OsPP2C50 induced the expression of GUS in rice guard cells in an ABA-dependent manner (Fig. 4d), whereas OsPP2C53 constitutively drove expression of GUS in rice guard cells regardless of
ABA treatment (Fig. 4c). RT-qPCR analysis confirmed that OsPP2C50 is predominantly expressed in shoots, whereas OsPP2C06 and OsPP2C53 are expressed in both shoots and roots (Fig. 4e, f, g). Expression of OsPP2C06 was not induced by ABA treatment. Without ABA treatment condition OsPP2C53 was expressed 30\% more than OsPP2C50, but in ABA treatment condition, OsPP2C50 was induced much strongly than that of OsPP2C53 (Fig. 4h).

OsPP2C50 and OsPP2C53 Physically Interact with SAPK10 and their Overexpression Lines Present Higher Water Loss in Detached Leaves and Drought Sensitivity

PP2CAs can physically interact with and negatively regulate SnRK2/SAPK activity in Arabidopsis and rice (Bhatnagar et al. 2017). Thus, we investigated whether OsPP2C50 and OsPP2C53 can physically interact with SAPK10. First, we performed yeast two-hybridization using SAPK10 as bait (BD-SAPK10) and nine OsPP2CAs as prey (AD-OsPP2CA). OsPP2C6, 50, 53 and 09 showed colony growth on selection media, indicating that they interacted with SAPK10, but other OsPP2CAs did not (Additional file 2: Figure S2). BiFC assays also revealed fluorescent signals between VN-SAPK10 and OsPP2C53-VC or OsPP $2 \mathrm{C} 50-\mathrm{VC}$ in rice protoplasts, but not between VN-SAPK10 and Sar1p-VC, which was used as negative control. The VN-SAPK10-OsPP2C53-VC and VN-SAPK10-OsPP2 C50-VC complexes were localized in the cytosol of rice protoplasts (Fig. 5a). For further interaction studies, we performed co-IP assays. SAPK10-Flag was detected when OsPP2C53-GFP or OsPP2C50-GFP were precipitated with an anti-GFP trap (Fig. 5b). Furthermore, it was confirmed that OsPP2C50 and OsPP2C53 could inhibit the phosphorylation function of SAPK10 in in vitro phosphorylation assay (Additional file 5: Figure S5). Taken together, OsPP2C53 and OsPP2C50 are expressed in rice guard cells and can interact with SAPK10 as negative regulators.

We also generated transgenic rice lines E25 and E40 overexpressing OsPP2C53 and OsPP2C50, respectively. Three over-expression lines were selected for water loss assays based on their gene expression levels (Additional file 4: Figure S4C and D). Water loss of the E25 and E40 lines was significantly higher than that of the control in all lines tested (Fig. 5c, d). In addition, the transgenic lines showed drought hypersensitivity at the young seedling stage (Additional file 3: Figure S3). Taken together, OsPP2C50 and OsPP2C53 might negatively regulate SAPK10 and OsSLAC1 in stomatal closure.

\section{OsPP2C50 and OsPP2C53 Directly Interact with the N- Terminal Region of OsSLAC1}

In Arabidopsis, AtPP2CA interacts with SLAC1 during ABA signaling, and ABI1 can directly dephosphorylate 

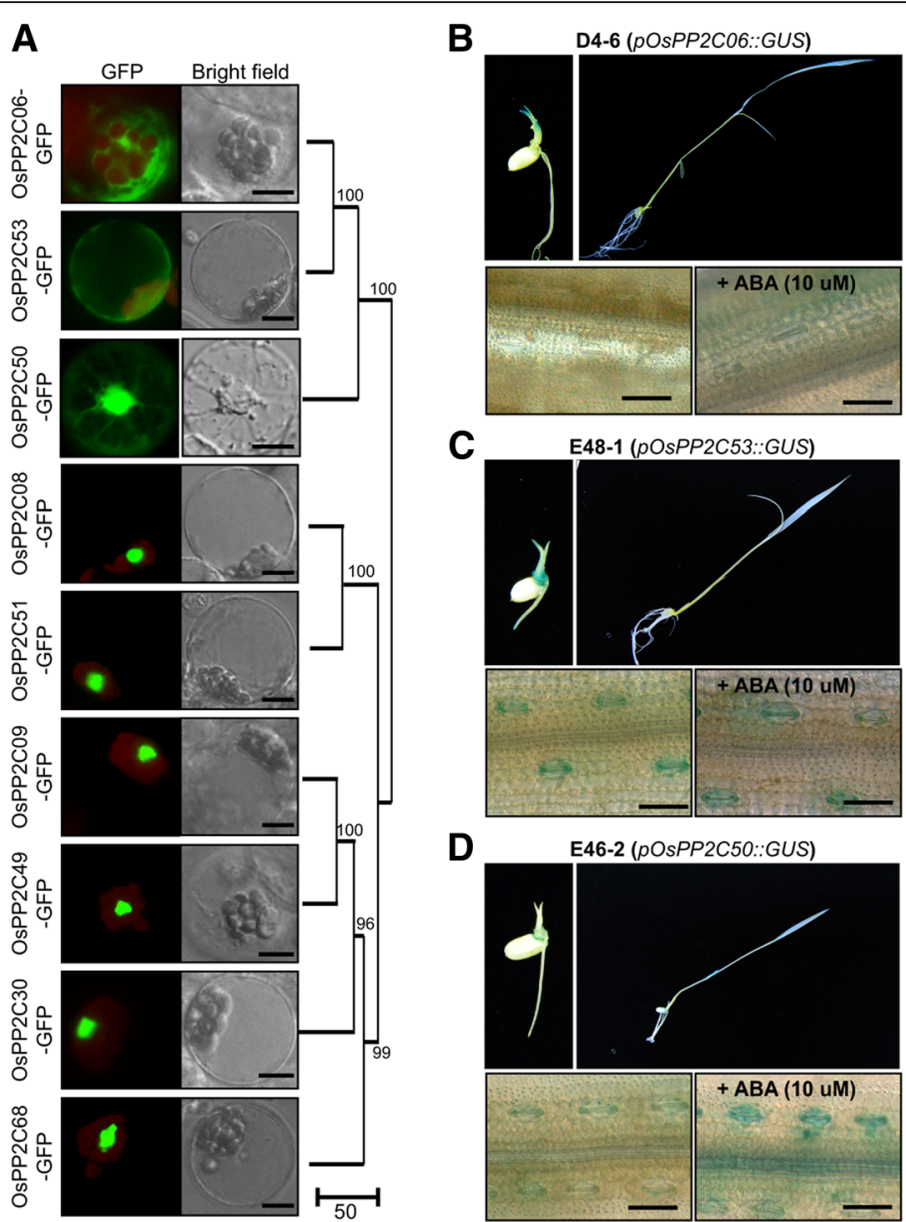

D E46-2 (pOsPP2C50::GUS)
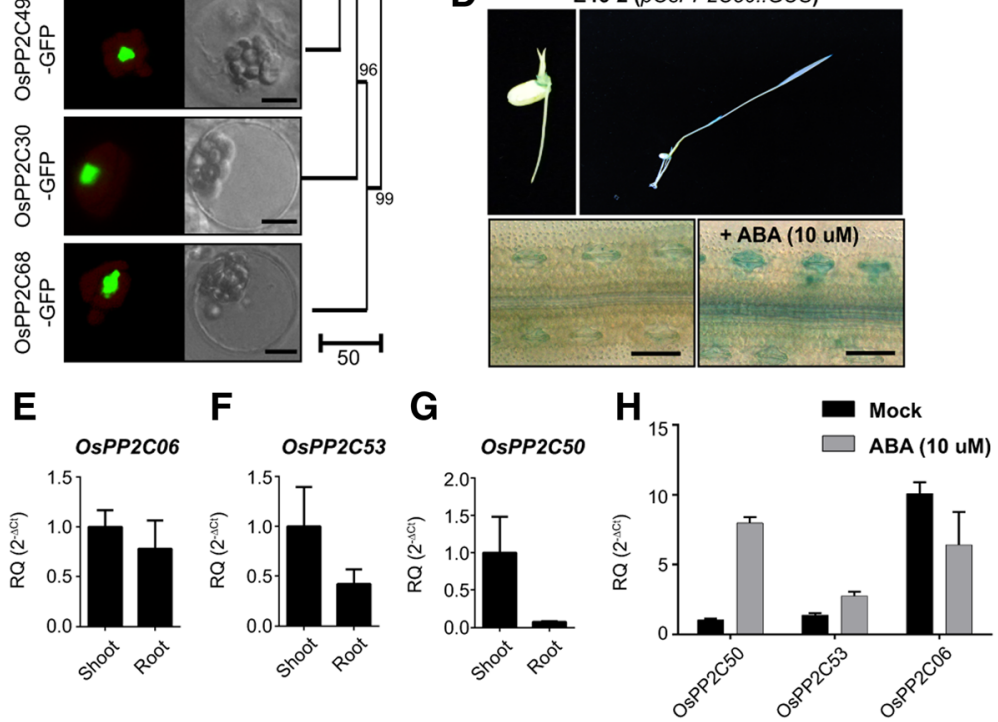

Fig. 4 Expression of OSPP2C50 and OSPP2C53 in rice guard cells and subcellular localizations of OsPP2CAs. a Subcellular localizations of nine OsPP2CAs were observed in rice protoplasts. Scale Bars $=10 \mu \mathrm{m}$. a Subcellular localization of OsPP2CA-GFPs in rice protoplasts. b, $\mathbf{c}, \mathbf{d}$ Histochemical $\beta$-glucuronidase analysis of OsPP2CA promoter-GUS transgenic rice. Leaf surface of transgenic rice harboring OsPP2C06 promoter-GUS (D4-6), OsPP2C53 promoter-GUS (E48-1), and OSPP2C50 promoter-GUS (E46-2) with or without ABA treatments were stained with X-Gluc. Scale bars are $50 \mu \mathrm{m}$. e, $\mathbf{f}, \mathbf{g}$ RT-qPCR analysis of OsPP2C06, OsPP2C50 and OsPP2C53 using two-week-old seedlings. The generated RQ (relative quantity, $2^{-\Delta C t}$ ) was compared to the value of expression on shoot. OsUbi5 was used as endogenous control. The values depict the average and \pm SD with 3 replications. $\mathbf{h}$ RT-qPCR analysis of OsPP2C06, OsPP2C50 and OsPP2C53 using two-week-old seedlings treated or not treated with ABA. The detected RQ (relative quantity, $2^{-\triangle C t}$ ) displayed OsUbi5 was used as endogenous control. The values depict average and \pm SD with 3 replications

the N-terminal region of SLAC1 even in response to $\mathrm{Ca}^{2}$ ${ }^{+}$signaling (Brandt et al. 2012; Geiger et al. 2009; Lee et al. 2009; Maierhofer et al. 2014).

Accordingly, we explored the physical interaction between OsPP2C50 and OsPP2C53 using Co-IP analysis. First, we identified that N-OsSLAC1-GFP can physically interact with OsPP2C50-HA and OsPP2C53-HA. Next, we identified the interaction motif of N-OsSLAC1 responsible for binding to OsPP2C50-HA and OsPP2C53-HA. OsPP2C50-HA and OsPP2C53-HA co-precipitated with $\mathrm{N}$-OsSLAC1-GFP, the N-terminal 25 amino acid deletion of OsSLAC1 $(\Delta 25)$, and N51-GFP, containing only the first 51 amino acids of OsSLAC1 (Fig. 6a). However, neither the $\Delta 35$ nor $\Delta 50 \mathrm{~N}$-terminal deletions were 

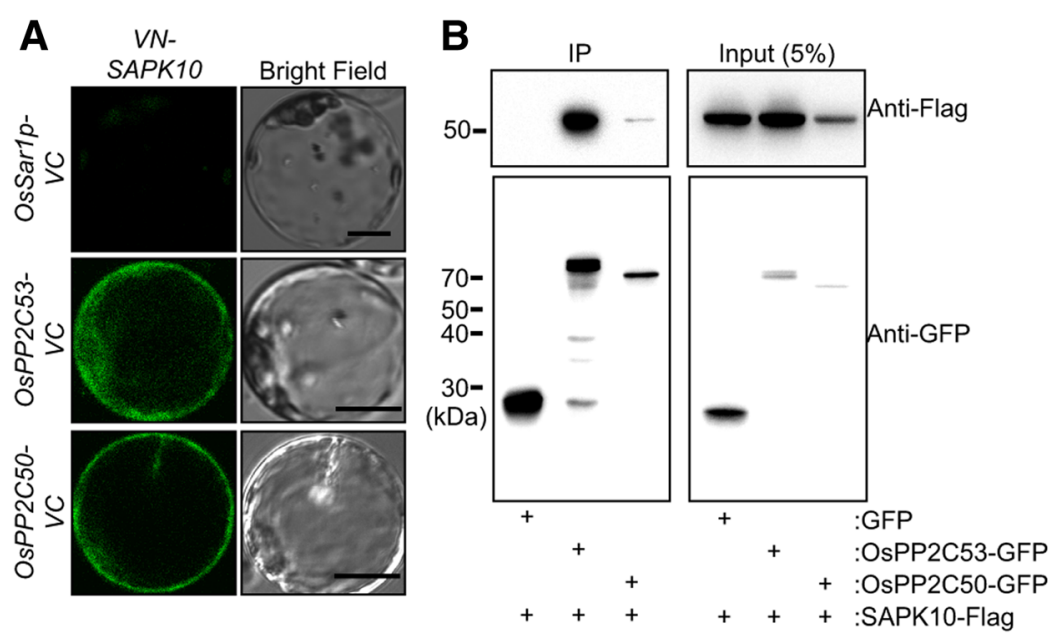

C

D
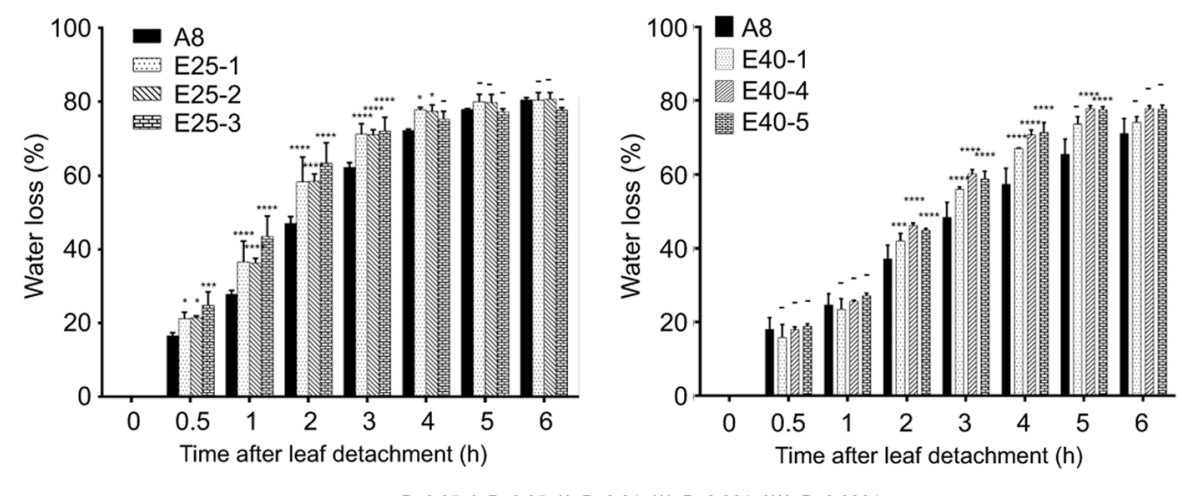

Fig. 5 OsPP2C50 and OsPP2C53 physically interact with SAPK10 and their overexpression promotes water loss. a BiFC analysis of VN-SAPK10 and OsPP2C53-VC or OsPP2C50-VC. OsSar1p-VC was used as a negative control. Scale bars are $10 \mu \mathrm{m}$. b Co-IP analysis using OsPP2C53-GFP or OsPP2C50-GFP and SAPK10-Flag expressed in rice protoplasts. These experiments were repeated three times and a representative result was presented. c Water loss assays of OSPP2C53-overexpressing transgenic lines (E25-1, 2 and 3) and control line (A8: empty vector transgenic plant). d Water loss assay of OSPP2C50-overexpressing transgenic lines (E40-1, 4 and 5) and control line (A8: empty vector transgenic plant). The values depict the average and \pm SD with 3 replications. ANOVA analyses were performed by comparing with water loss of A8 plant.

co-precipitated. These results revealed that the N-terminal 26-35 amino acids of OsSLAC1, which are predicted to form the second alpha-helix in the $\mathrm{N}$-terminal region of OsSLAC1 (Fig. 6b), play an important role in the interaction with OsPP2C50 and OsPP2C53.

\section{Discussion}

More than $95 \%$ of water is evaporated through stomatal pores in plants. Stomatal movement therefore has crucial functions in the water usage efficiency of plants (Schroeder et al. 2001). Thus far, the molecular mechanisms for stomatal closure and opening have been most studied in the model plant Arabidopsis, whereas studies in monocot plants, including most crops such as rice, maize, wheat and barley, have been limited (Chen et al. 2017; Hwang et al. 2013; Moon et al. 2017; Raissig et al. 2017; Sun et al. 2016). In this study, we unraveled the core ABA signaling components and networks functioning in stomatal closure of rice, a model monocot plant. Our systematic and comparative functional analysis suggests that rice and Arabidopsis have evolutionally conserved ABA signaling components, which interact in similar interaction networks, for stomatal closure, despite their stomatal complexes having different anatomical structures.

\section{ABA Signaling Components Functioning in Stomatal Closure are Evolutionally Conserved Between Monocots and Dicots}

Earlier studies have identified SLAC1 homologs in Arabidopsis, rice, and barley based on their amino acid similarity and genetic analysis (Negi et al. 2008; Saji et al. 2008; Vahisalu et al. 2008). OsSLAC1 is the most similar to Arabidopsis SLAC1 of the rice OsSLAHs 


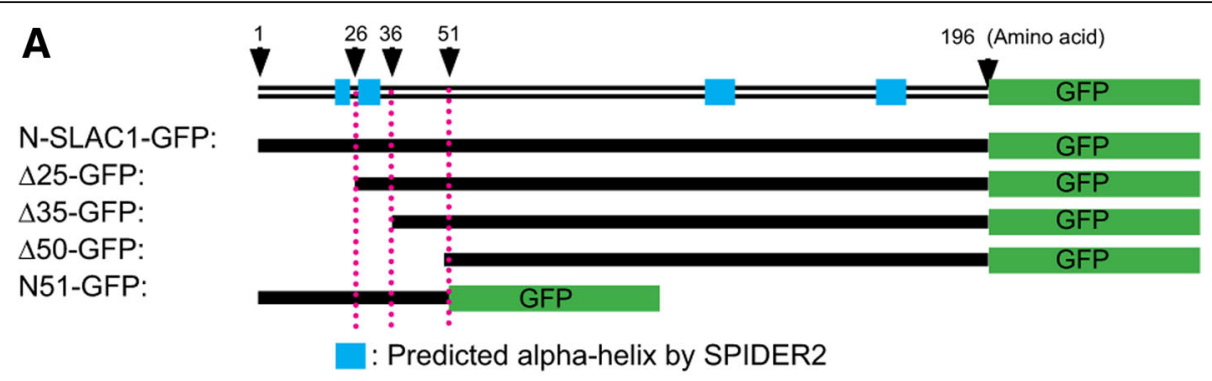

B

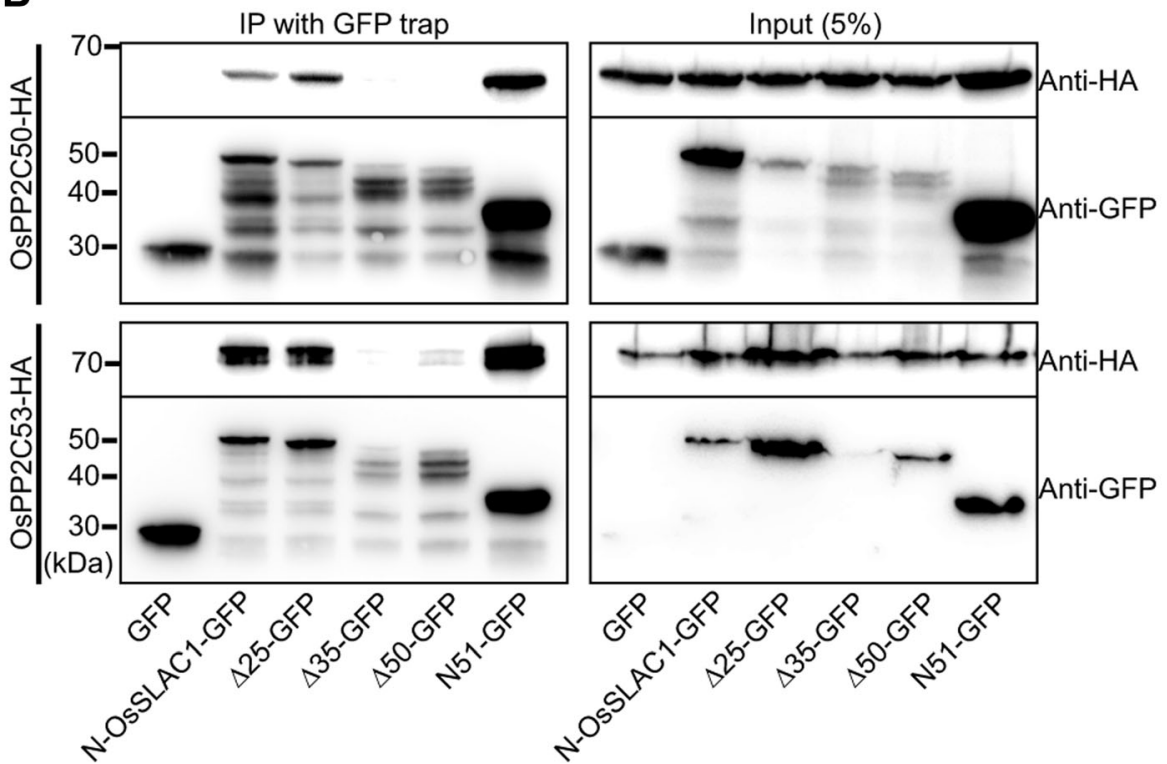

Fig. 6 OsPP2C50 and OsPP2C53 directly interact with OsSLAC1-N-terminal region. a Diagram of deletion mutant constructs of OsSLAC1 used for Co-IP. $\mathbf{b}$ Co-IP analysis using OsPP2C50-HA and several deletion mutant forms of N-SLAC1-GFP co-expressed in rice protoplasts. These experiments were repeated three times and a representative result was presented

(Vahisalu et al. 2008). We additionally demonstrated that OsSLAC1 was specifically expressed in rice guard cells, in contrast to the other OsSLAHs. Even though the SLAC1 proteins of monocot and dicot plants have highly conserved amino acid sequences, they have different electrophysiological characteristics. Monocot SLAC1 requires extracellular nitrate to activate it under ABA whereas SLAC1 from dicots does not (Hedrich and Geiger 2017; Schafer et al. 2018). Also monocots and dicots have different anatomical structure in stomatal complex which monocots consists of a pair of dumbbell-shaped guard cells and a pair of subsidiary cells, in contrast to the kidney-shaped guard cells typical of dicots. Accordingly, we thought that the ABA signaling components, pathways and molecular mechanisms functioning in the guard cells of monocots and dicots might differ.

Here, we identified signaling networks consisting of SAPK10, OsPP2C50 and OsPP2C53 as the core ABA signaling components related to the regulation of
OsSLAC1 in rice guard cells. We demonstrated that these genes correspond to Arabidopsis OST1, ABI1 and $A B I 2$, based on phylogenetic analysis, guard cell-specific gene expression, subcellular localization, and physical interaction and water loss assays of transgenic plants overexpressing those genes. Taken together, the core ABA signaling components functioning in guard cells are highly conserved in terms of gene functions, expression profiles, interaction and structures in monocots and dicots. Thus, the molecular mechanisms of ABA signaling in guard cells might be conserved among monocots and dicots, although further studies regarding these components might discern differences in the mechanistic details of the two.

\section{Clade A PP2Cs Localized Ubiquitously in Cells Might Play Roles in Diverse Signaling Pathways}

In our results nine OsPP2CAs can be divided into two groups based on their subcellular localization patterns. One is confined in nuclei and the other is localized 
ubiquitously in the cell, including nuclei, cytosol and ER. Similar subcellular localization patterns have also been observed in Arabidopsis (Umezawa et al. 2009). Thus, the subcellular localization patterns of PP2CAs might be functionally important and evolutionary conserved in rice and Arabidopsis. Proteins that interact with PP2CAs and signaling molecules are differentially distributed in the subcellular organelles. For example, OsPP2C51 which is localized specifically in nuclei can interact with nuclear localized transcription factor OsbZIP 10 (Bhatnagar et al. 2017). ABI1, known to be localized in the cytosol, can interact with the $\mathrm{N}$-terminal region of AtSLAC1 that protrudes into the cytosol in Arabidopsis (Brandt et al. 2012; Lee et al. 2009). It was known that stomatal closure and opening are affected by diverse environmental cues such as $\mathrm{ABA}, \mathrm{Ca}^{2+}, \mathrm{CO} 2$ and $\mathrm{H}_{2} \mathrm{O}_{2}$ and by signaling components such as SnRK2, CIPK, CDPK, and MAPKs. OsPP2C50 and OsPP2C53 among OsPP2CAs might be localized ubiquitously in cell such as the cytosol, ER, nuclei or plasma membrane. Thus they might interact with diverse signaling components and play a role as common negative regulators of stomatal opening in different signaling. That is one of the reasons why plant overexpressing them showed significant drought sensitivity and water loss phenotype. It also show that OsPP2C50 and OsPP2C53 are common negative regulator of stomata closure signaling that the interaction domain of N-terminal OsSLAC1 is close to amino acid S59 which is similar position S50 of AtSLAC1 phosphorylated by CDPKs. Two OsPP2Cs interacted at similar position of N-terminal OsSLAC1 (25-36) could inhibit the phosphorylation function of SAPK10, which could phosphorylate the N75-OsSLAC1 (Additional file 5: Figure S5).

Differences between OsPP2C50 and OsPP2C53 in terms of ABA-dependent gene expression suggest they might have different functions in $\mathrm{ABA}$ signaling. Without ABA condition both OsPP2C50 and OsPP2C53 might function as suppression components in stomata closure and under the high ABA condition the ABA-dependently expressed OsPP2C50 might play a role as a major negative inhibitor in guard cell closure.

One of the biggest challenges facing agricultural researchers is to improve water usage efficiency of crops because the cereal supply for humans is threatened by water deficiency caused by global warming. Studies on stomatal movement regulation are a major research target for improving water use efficiency because stomatal pores are nearly the only gate by which water leaves the plant. In this study, we identified two PP2CAs, OsPP2C50 and OsPP2C53 as very strong negative regulators of stomatal closure functioning in rice guard cells. Thus, these two genes might be promising targets for engineering improved water use efficiency in monocots.

\section{Conclusion}

In this study we found that OsPP2C50 and OsPP2C53 specifically expressed in rice guard cell and is major negative regulators of $\mathrm{ABA}$ signaling regarding stomata closing in rice. OsPP2C50 and 53 can interact with the N-terminal of OsSLAC1 directly or can interact with SAPK10 which is specifically expressed in rice guard cell.

\section{Materials and Methods \\ Plant Growth Conditions and Generation of Transgenic Plants}

Husked rice seeds (Oryza sativa subsp. japonica cv. Dongjin) were sterilized by immersion in $70 \%$ ethanol for $30 \mathrm{~s}$, and in $2 \% \mathrm{NaClO}$ for $40 \mathrm{~min}$. After washing 5 times with distilled water, the sterilized seeds were planted on half-strength (1/2) Murashige and Skoog (MS; Duchefa Biochemie, Netherlands) medium supplemented with 0.4\% phytagel and adjusted to $\mathrm{pH} 5.8$. The plants were grown in a culture room at $28^{\circ} \mathrm{C}$ under $16 / 8 \mathrm{~h}$ light/dark cycles.

$1000 \sim 1500$ bp upstream regions of each of OsSLAC1, SAPK10, OsPP2C06, OsPP2C53, and OsPP2C50 were isolated from rice genomic DNA by polymerase chain reaction (PCR) amplification with the primers indicated in Additional file 8: Table S1 to generate the transgenic plants harboring promoter-GUS reporters. The PCR products were cloned using the pENTR/D-Topo cloning kit (Invitrogen, USA), and transferred to the promoter region of the pBGWSF7 vector, a GUS-expressing binary vector, via an LR recombination reaction using the Gateway system (Invitrogen, USA) (Karimi et al. 2002). The full-length cDNAs were cloned using the pENTR/ D-Topo cloning kit and then transferred into the pGA2897 vector harboring the Maize UBIQUITIN promoter (Kim et al. 2012). The resulting plasmids were transferred into Agrobacterium competent cells (Agrobacterium tumefaciens strain LBA4404). Rice transgenic plants were generated using the Agrobacterium-mediated co-cultivation method (Han et al. 2012; Toki et al. 2006). Transformants were screened based on phosphinothricin (PPT) resistance and hygromycin resistance for pBGWFS7 and pGA2897, respectively.

\section{Total RNA Isolation and Reverse Transcription Quantitative PCR}

Total RNA was isolated from five-day-old rice seedlings for over-expression line selection and from two-week-old rice seedlings and mature plants for identifying expression levels using the Qiagen RNeasy Plant Mini Kit (QIAGEN, USA). Contaminating genomic DNA was digested with Recombinant DNase 1 (RNase-free) (TAKARA BIO, Japan). From total RNA ( $3 \mu \mathrm{g})$, first strand cDNA was synthesized with oligo $\mathrm{dT}$ and random hexamers using the SuperScript ${ }^{\mathrm{sm}}$ Reverse Transcriptase first strand synthesis 
system (Invitrogen, USA) following the manual. Reverse transcription quantitative PCR (RT-qPCR) assays using the $\mathrm{iQ}^{\mathrm{tm}} \mathrm{SYBR}^{\circ}$ Green Supermix (Biorad, USA) were employed in this study.

The reaction mixture was subjected to denaturation at $95^{\circ} \mathrm{C}$ for $3 \mathrm{~min}$, followed by $30 \sim 40$ cycles of denaturation at $94{ }^{\circ} \mathrm{C}$ for $10 \mathrm{~s}$, annealing at $58^{\circ} \mathrm{C}$ for $15 \mathrm{~s}$, and elongation at $72{ }^{\circ} \mathrm{C}$ for $20 \mathrm{~s}$. Triplicate quantitative assays were performed on each cDNA sample. Sequences of the primers used are listed in Additional file 8: Table S1. RT-qPCR was performed with internal primers, $U B I 5$ was used as the control, and relative gene expression was analyzed by the delta-delta Ct method (Dooms et al. 2014).

\section{Rice Protoplast isolation and PEG-Mediated Transient Transformation}

To isolate rice protoplasts, plants were germinated and grown for 10 days on $1 / 2$ MS medium supplemented with $0.4 \%$ phytagel adjusted to $\mathrm{pH} 5.8$ and then subjected to 8 days in the dark followed by 2 days under a $16 / 8 \mathrm{~h}$ light/dark cycle at $28^{\circ} \mathrm{C}$ in an incubation room. The resulting plants were chopped into approximately $1 \mathrm{~mm}$ pieces and immediately transferred into freshly prepared enzyme solution ( $1.5 \%$ cellulose $\mathrm{R}-10,0.75 \%$ Macerozyme R-10, $0.6 \mathrm{M}$ mannitol, $10 \mathrm{mM} \mathrm{CaCl} 2,0.1 \%$ bovine serum albumin (BSA), $0.06 \% \beta$-mercaptoethanol and 10 $\mathrm{mM}$ 2-(N-morpholino) ethanesulfonic acid at $\mathrm{pH} 5.7$ ). After gentle shaking at $60-80 \mathrm{rpm}$ for $4 \mathrm{~h}$ at room temperature, protoplasts were isolated by passing through a $100 \mu \mathrm{m}$ diameter mesh and sedimented by centrifugation at $100 \times g$. After washing with W5 solution $(154 \mathrm{mM} \mathrm{NaCl}, 125 \mathrm{mM} \mathrm{CaCl}, 5 \mathrm{mM} \mathrm{KCl}$, and $2 \mathrm{mM}$ MES adjusted to $\mathrm{pH} 5.7)$, isolated protoplasts were resuspended in MaMg solution (0.6 M mannitol, $15 \mathrm{mM}$ $\mathrm{MgCl}_{2}$, and $5 \mathrm{mM} \mathrm{MES}$ at pH 5.7) at a concentration of $3-5 \times 10^{6}$ cells $\mathrm{mL}^{-1}$. Then, $0.3 \mathrm{~mL}$ of suspended cells was gently mixed together with the DNA plasmids indicated and freshly prepared $0.33 \mathrm{~mL}$ polyethylene glycol electrolyte (PEG) solution $(40 \%(w / v)$ polyethylene glycol (MW 6000), 0.1 $\mathrm{M} \mathrm{CaNO}_{3}$, and 0.4 M mannitol). After $25 \mathrm{~min}$ incubation at room temperature, the samples were serially diluted with W5 solution $(630 \mathrm{~mL}$, $1200 \mathrm{~mL}$ and $2500 \mathrm{~mL}$ ) and mixed for $10 \mathrm{~min}$ each time. Finally, the protoplasts were incubated with W5 solution at $28^{\circ} \mathrm{C}$ until use (Kim et al. 2015).

\section{Subcellular Localization and BiFC}

To observe subcellular localization of GFP-fused proteins, the coding sequences (CDS) of selected genes were inserted into pENTR/D-topo vectors (Invitrogen, CA, USA). CDSs were transferred into pMDC43 and pMDC83 vectors (Curtis and Grossniklaus 2003) via LR recombination reactions using the Gateway system (Invitrogen, CA,
USA) (Curtis and Grossniklaus 2003). After purification, the constructs were introduced into rice protoplasts using a PEG-mediated method. GFP and mCherry signals were captured using a Leica TCS SP8 laser scanning confocal microscope and fluorescence microscopy (Carl Zeiss, DE/ Axio imager M1). The combinations of excitation wavelength / detection range of emission on the confocal microscopy were $488 \mathrm{~nm}$ (solid state laser)/ 493 to 530 bandpass for GFP and $552 \mathrm{~nm}$ (solid state laser)/ 603 to 662 bandpass for mCherry. For fluorescence microscopy, filter sets were XF116 (exciter, 474AF20; dichroic, 500DRLP; emitter, 510AF23) for GFP and XF33/E (exciter, 535DF35; dichroic, 570DRLP; emitter, 605DF50 [Omega]) for mCherry. The detected images are presented in pseudocolor. To quantify the rate of co-localization between two different proteins on an image, images were analyzed by the Leica Application SuitX analysis program with default settings (threshold 30\%, background 20\%).

To observe interactions between proteins using bimolecular fluorescence complementation (BiFC), inserts were introduced into pENTR-D-TOPO vectors (Invitrogen, $\mathrm{CA}$ ) and then transferred to their destination vectors (pGEM-gw-VC or pGEM-VC-gw vectors for Venus Carboxyl-terminus (VC) tagging, and pGEM-VN-gw or pGEM-gw-VN vectors for Venus Amino-terminus (VN) tagging using LR recombination (Promega, WI, USA). All BiFC gateway vectors (pGEM-gw-VN, pGEM-VN-gw, pGEM-gw-VC, and pGEM-VC-gw) were prepared to amplify Gateway cassette regions (35S promoter to Nos-terminator) of pDEST-VYNE, pDEST-VYNE (R), pDEST-VYCE, and PDEST-VYCE(R) by PCR reaction with primers (5'-HindIII-35S and 3'-EcoRI-nos) and ligate with the pGEM-T Easy vector (Promega, WI, USA) (Gehl et al. 2009). Fluorescence signals were captured using a Leica TCS SP8 laser scanning confocal microscope. The combination of excitation wavelength / detection range of emission for Venus signals was $488 \mathrm{~nm}$ (solid state laser)/ 505 to 561 bandpass. Signal intensities on captured images were analyzed using Leica Application SuiteX.

\section{In Vitro Phosphorylation Assay}

In vitro phosphorylation assays of SAPK10 activity on OsSLAC1 were carried out as previously described (Bhatnagar et al. 2017; Chae et al. 2007). The CDSs of SAPK10 and the N-terminal region of OsSLAC1 were cloned into pRST-A and pGEX-5x-1, respectively. 6xHis-tagged SAPK10 and GST-tagged proteins (NOsSLAC1 and N-OREB1) were purified using Ni-NTA resins (Thermo Scientific, IL, USA) and Glutathione Sepharose High Performance resins (GE Healthcare, Sweden), respectively. Aliquots of His-fused SAPK10 kinase and substrates were incubated in phosphorylation buffer $(10 \mu \mathrm{Ci} \gamma$-32P-ATP, $10 \mu \mathrm{M}$ ATP, $20 \mathrm{mM}$ Tris-HCl $\mathrm{pH}$ 7.0, $5 \mathrm{mM} \mathrm{MgCl} 2$ or $\mathrm{MnCl}_{2}$ ) for $30 \mathrm{~min}$ at $30^{\circ} \mathrm{C}$. After 
stopping the reaction by addition of $10 \mathrm{mM}$ EDTA, the products were analyzed by sodium dodecyl sulfate polyacrylamide gel electrophoresis (SDS-PAGE). Gels were stained with Coomassie Brilliant Blue R-250, dried, and analyzed with a phosphoimage analyzer (Personal Molecular Imager FX system, Bio-Rad, Benicia, CA, USA).

\section{Histochemical GUS Expression Analysis}

To test gene expression, seedlings or mature GUS-expressing transgenic plants were stained with 5-Bromo-4-chloro-3-indolyl- $\beta$-D-glucuronide (X-Glu), a substrate of GUS. Plant materials were briefly washed with $90 \%$ acetone, then analyzed following the methods previously reported using GUS-staining buffer $[2 \mathrm{mM}$ X-Gluc, $50 \mathrm{mM} \mathrm{NaSO} 4,0.1 \%$ Triton X-100, $0.5 \mathrm{mM}$ $\mathrm{K}_{3} \mathrm{Fe}^{\mathrm{III}}(\mathrm{CN})_{6}$, and $0.5 \mathrm{mM} \mathrm{K} \mathrm{Fe}^{\mathrm{II}}(\mathrm{CN})_{6}$, adjusted to $\mathrm{pH}$ 7.0] (Kim et al. 2015). GUS-stained plants and tissues were fixed by washing several times with $70 \%$ ethanol until the chlorophyll was completely removed from the tissue. Paraffin sectioning and eosin staining were completed by conventional methods. In brief, samples were embedded in paraffin and $4 \mu \mathrm{m}$ cross-sections were made using a RM2255 microtome (Leica, Germany). These slices were stained with eosin following standard manual, mounted with Permount (Thermo Fisher Scientific, USA) and observed using a Zeiss AxioCam MRc CCD camera (Carl Zeiss, Jena, Germany).

\section{Co-Immunoprecipitation Assay}

To perform immunoprecipitation experiments, we used GFP-trap (Chromotek, Germany) for the immunoprecipitation of GFP-fusion proteins. All tested genes were inserted into pENTR/D-topo vectors (Invitrogen, CA, USA) and then recombined with pGEM-gw-GFP or pGEM-GFP-gw vectors for GFP fusion, pGEM-gw3xHA vector for HA tagging, and pGEM-gw-Flag vector for Flag tagging using LR recombinase (Invitrogen, CA, USA). To generate pGEM-gw-GFP or pGEM-GFP-gw vectors, the Gateway cassette regions (35S promoter to Nos-terminator) of pMDC83 and pMDC43 were amplified by PCR with primers (5-pMDC and 3-pMDC), and the PCR products were inserted into pGEM-T easy vectors (Promega, WI, USA). To generate pGEM-gw-3xHA and pGEM-gw-Flag vectors, the Gateway cassette regions (Ubi promoter to nos terminator) of pGA2897were amplified by PCR with primers (5-Ubi and $3-n o s-t)$, and then the PCR products were ligated with the pGEM-T easy vector (Promega, WI, USA). The indicated constructs were introduced into rice protoplasts using the PEG-mediated method, and the transformed protoplasts were incubated at $28^{\circ} \mathrm{C}$. Cellular extracts from transformed protoplasts in immunoprecipitation buffer $[150 \mathrm{mM} \mathrm{NaCl}, 50 \mathrm{mM}$ Tris- $\mathrm{HCl}$ at $\mathrm{pH} 7.5,1 \mathrm{mM}$ EDTA, $2 \mathrm{mM}$ EGTA, $2 \mathrm{mM} \mathrm{MgCl}_{2}, 0.5 \% \mathrm{NP} 40,0.5 \%$
Triton X-100, and $1 \mathrm{x}$ protease inhibitor cocktail (complete ULTRA tablet, Roche, IN, USA)] were incubated with pre-cleaned GFP-trap beads at $4{ }^{\circ} \mathrm{C}$ for $2 \mathrm{~h}$. After washing 5 times with immunoprecipitation buffer, the precipitated proteins, together with GFP-trap, were subjected to SDS-PAGE and immunoblot analysis. Precipitated GFP, HA, or Flag-tagged proteins were detected with anti-GFP rabbit antibody (Life Technologies, OR, USA), anti-HA rat antibody (Roche, IN, USA), or anti-Flag M2 peroxidase antibody (Sigma), respectively.

\section{Yeast Two-Hybrid Assay}

Yeast two-hybridization assays were performed using the Matchmaker $^{\mathrm{Tm}}$ GAL4 Two-Hybrid System 3 (Clontech, USA) according to the manufacturer's manual. The lithium acetate method was used to introduce pGADT7 (as the yeast activation domain) and pGBKT7 (as the yeast binding domain) plasmids into yeast strain Saccharomyces cerevisiae strain AH109. Yeast were plated on Yeast Minimal Media/Synthetic Defined (SD) media (Clontech, USA) without leucine and tryptophan, then transferred to selection media without leucine, tryptophan and histidine supplemented with $15 \mathrm{mM}$ 3-amino-1, 2, 4-triazole (3-AT) (Sigma, USA). For yeast spotting assays, exponentially grown yeast cells were harvested and adjusted to $\mathrm{OD}_{600} 0.5$ with sterilized water and diluted $1 / 10$ and $1 / 100$. Yeast cells were spotted onto SD medium without leucine and tryptophan and SD medium without leucine, tryptophan and histidine. Growth was assessed 3 days after spotting.

\section{Water Loss Assay}

For water loss of detached leaves, leaves were removed from A8 (pGA2897 empty vector) transgenic plants as a control and OsSLAC1, SAPK10 and OSPP2CA50 and OsPP2CA53 over-expression lines that had been grown for 10 days in a square box $(72 \mathrm{~mm} \mathrm{X} 72 \mathrm{~mm} \mathrm{X} 100$ $\mathrm{mm}$ ) with $1 / 2 \mathrm{MS}$ medium under long day conditions (16 h light and $8 \mathrm{~h}$ dark) at $28^{\circ} \mathrm{C}$ in a cultivation room. One day before measurements, the box cover was opened, and plants were allowed to adapt to outside conditions. Detached leaves were placed on a laboratory bench and periodically weighed. The experiment was performed each time with three replicate leaves per line.

\section{Drought Assay}

For drought stress tolerance analysis, seeds from OsPP2CA53 over-expression lines (E25) and OsPP2CA50 over-expression lines (E40) were grown in white square pots with soil in a growth chamber with sufficient water until the fourth leaf stage. Each sample was tested in three independent experiments. Watering was stopped for 5 days until leaves completely wilted just before dying, and then the plants were re-watered. After 
recovery of the plants, the survival rates of the plants were measured.

\section{Additional Files}

Additional file 1: Figure S1. OSSLAC1 has the highest homology to AtSLAC1 and is expressed predominantly in leaf tissues. (A) Phylogenetic tree depicting S-type anion channels of Arabidopsis and their homologues in rice. This tree was constructed using the neighbor-joining method in Mega 6.0. (B) Tissue-specific expression data for rice S-type anion channels were analyzed using the Rice X pro database. (TIF $3251 \mathrm{~kb}$ )

Additional file 2: Figure S2. Yeast two hybridization assay between BDSAPK10 and AD-OsPP2CAs. SD: Synthetic Drop-out medium, L: leucine, T: Tryptophan, H: Histidine, 3AT: 3-amino-1,2,4-triazole. (TIF 1196 kb)

Additional file 3: Figure S3. Transgenic rice overexpressing OsPP2C50 and OSPP2C53 present drought hypersensitive phenotype. Pictures showing drought hypersensitivity of OSPP2C53-overexpressing transgenic lines (E25-1, 2 and 3) compared to control line (DJ, Dong Jin) (A) and OsPP2C50-overexpressing transgenic lines (E40-1, 4 and 5) compared to control line $(C)$. ( $B, D)$ Survival rate after rewatering the drought treated rice of each transgenic line. These data obtained from 3 independent experiment, $N>20$. The values depict the average and \pm SD. (TIF $6522 \mathrm{~kb}$ )

Additional file 4: Figure S4. Confirmation of gene overexpression in transgenic rice. All overexpressing transgenic plants were confirmed by RTqPCR analysis with each specific primer. The expression level of Ubiqutin5 was used by endogenous control. All values were compared to the value of DJ. (A) OsSLAC1, (B) SAPK10, (C) OsPP2C53, and (D) OsPP2C50. (TIF 1919 kb)

Additional file 5: Figure S5. Inhibition of kinase activity of SAPK10 by OsPP2C50 or OsPP2C53 In vitro kinase assay were performed with OsPP2C50 or OsPP2C53. Each protein were used indicated quantity (below) and phosphorylated with SAPK10 in reaction buffer $(60 \mathrm{mM} \mathrm{NaCl}$, $20 \mathrm{mM}$ Tris-Cl, pH 7.4, $5 \mathrm{mM} \mathrm{MgCl}, 10 \mu \mathrm{M}$ ATP, and $10 \mu \mathrm{Ci}$ Y-32P-ATP) at $30{ }^{\circ} \mathrm{C}$ for $30 \mathrm{~min}$. (A) Detected phosphor image, (B) Polyacrylamide gel stained with Coomassie brilliant blue R250. (TIF $2460 \mathrm{~kb}$ )

Additional file 6: Figure S6. The expression of SAPKs, OsPP2Cs and OsSLAC1/OsSLAHs in anatomy microarray data base. (A,B,C) OsSLAC 1/ OsSLAHs, OsPP2Cs, and SAPKs expression were search in microarray data base (Genevestigator). (D) The experimental ID (ex_ID) and used GEO numbers of DATA sets. (TIF $5518 \mathrm{~kb}$ )

Additional file 7: Supplementary methods. Purification of GST-N75OsSLAC1. To obtain the GST-N75-OsSLAC1, we constructed pGEX-5X-N75OsSLAC1. N-terminal region (N75) of OSSLAC1 was amplified by PCR reaction with specific primers (Additional file 8: Table S1) and harbored in pGEX-5x-1 vector with digestion by restriction enzymes (BamH1 and EcoR1) and ligation. Expressed GST-N75-OsSLAC1 in E. coli (BL21 pLysS) was purified using Glutathione Sepharose high performance (GE healthcare). (DOCX $13 \mathrm{~kb}$ )

Additional file 8: Table S1. Primers used in this manuscript. (XLSX 12 kb)

\section{Abbreviations}

3-AT: 3-amino-1, 2, 4-triazole; ABA: Abscisic acid; Arabidpsis: Arabidopsis thaliana; CIPK: CBL-INTERACTING PROTEIN KINASEs; CPK: CALCIUMDEPENDENT PROTEIN KINASE; GORK: K+outward-rectifying channel; GUS: $\beta$ glucuronidase; KAT1: $\mathrm{K}^{+}$-inward-rectifying channel; MS: Murashige and Skoog; OST1: OPEN STOMATA 1; PCR: Polymerase chain reaction; PEG: Polyethylene glycol electrolyte; PPT: Phosphinothricin; SD: Synthetic Defined; SLAC1: Slowtype anion channel; SnRK2: SNF1-RELATED PROTEIN KINASE2

\section{Acknowledgments}

Not applicable.

\section{Funding}

This work was supported by the Agenda Program (PJ01247602) and the Next Generation BioGreen 21 Program (PJ01367601) of the Rural Development Administration to Beom-Gi Kim.

\section{Availability of Data and Materials}

All data supporting the conclusions of this article are provided within the article and its Additional files 1, 2, 3, 4, 5, 6, 7 and 8 .

\section{Authors' Contributions}

MKM, EHC, JAK, SSH and YML performed the experiments. MKM, EHC, ISY, SHL, BGK analyzed the results. MKM, EHC and BGK designed the experiments and wrote the manuscript. All authors read and approved the final manuscript.

\section{Ethics Approval and Consent to Participate}

Not applicable.

\section{Consent for Publication}

Not applicable.

\section{Competing Interests}

The authors declare that they have no competing interests.

\section{Publisher's Note}

Springer Nature remains neutral with regard to jurisdictional claims in published maps and institutional affiliations.

\section{Author details}

${ }^{1}$ Metabolic Engineering Division, Department of Agricultural Biotechnology, National Institute of Agricultural Sciences, Rural Development Administration, Jeonju 54875, Republic of Korea. ${ }^{2}$ Gene Engineering Division, Department of Agricultural Biotechnology, National Institute of Agricultural Sciences, Rural Development Administration, Jeonju 54875, Republic of Korea. ${ }^{3}$ Department of Biological Sciences, Sungkyunkwan University, Suwon 16419, Republic of Korea.

Received: 11 November 2018 Accepted: 14 May 2019

Published online: 27 May 2019

\section{References}

Ache P, Becker D, Ivashikina N, Dietrich P, Roelfsema MR, Hedrich R (2000) GORK, a delayed outward rectifier expressed in guard cells of Arabidopsis thaliana, is a $\mathrm{K}(+)$-selective, $\mathrm{K}(+)$-sensing ion channel. FEBS Lett 486(2):93-98

Anderson JA, Huprikar SS, Kochian LV, Lucas WJ, Gaber RF (1992) Functional expression of a probable Arabidopsis thaliana potassium channel in Saccharomyces cerevisiae. Proc Natl Acad Sci U S A 89(9):3736-3740

Bauer H, Ache P, Lautner S, Fromm J, Hartung W, Al-Rasheid KA et al (2013) The stomatal response to reduced relative humidity requires guard cellautonomous ABA synthesis. Curr Biol 23(1):53-57. https://doi.org/10.1016/j. cub.2012.11.022

Bhatnagar N, Min MK, Choi EH, Kim N, Moon SJ, Yoon I et al (2017) The protein phosphatase 2C clade a protein OsPP2C51 positively regulates seed germination by directly inactivating OsbZIP10. Plant Mol Biol 93(4-5):389401. https://doi.org/10.1007/s11103-016-0568-2

Brandt B, Brodsky DE, Xue S, Negi J, Iba K, Kangasjarvi J et al (2012) Reconstitution of abscisic acid activation of SLAC1 anion channel by CPK6 and OST1 kinases and branched ABI1 PP2C phosphatase action. Proc Natl Acad Sci U S A 109(26):10593-10598. https://doi.org/10.1073/pnas. 1116590109

Chae MJ, Lee JS, Nam MH, Cho K, Hong JY, Yi SA et al (2007) A rice dehydrationinducible SNF1-related protein kinase 2 phosphorylates an abscisic acid responsive element-binding factor and associates with $A B A$ signaling. Plant Mol Biol 63(2):151-169. https://doi.org/10.1007/s11103-006-9079-x

Chen ZH, Chen G, Dai F, Wang Y, Hills A, Ruan YL et al (2017) Molecular evolution of grass stomata. Trends Plant Sci 22(2):124-139. https://doi.org/10. 1016/j.tplants.2016.09.005

Curtis MD, Grossniklaus U (2003) A gateway cloning vector set for highthroughput functional analysis of genes in planta. Plant Physiol 133(2):462469. https://doi.org/10.1104/pp.103.027979

Dooms M, Chango A, Abdel-Nour A (2014) Quantitative PCR (qPCR) and the guide to good practices MIQE: adapting and relevance in the clinical biology context. Ann Biol Clin (Paris) 72(3):265-269. https://doi.org/10.1684/abc.2014.0955

Gehl C, Waadt R, Kudla J, Mendel RR, Hansch R (2009) New GATEWAY vectors for high throughput analyses of protein-protein interactions by bimolecular fluorescence complementation. Mol Plant 2(5):1051-1058. https://doi.org/10. 1093/mp/ssp040 
Geiger D, Scherzer S, Mumm P, Marten I, Ache P, Matschi S et al (2010) Guard cell anion channel SLAC1 is regulated by CDPK protein kinases with distinct Ca2+ affinities. Proc Natl Acad Sci U S A 107(17):8023-8028. https://doi.org/ 10.1073/pnas.0912030107

Geiger D, Scherzer S, Mumm P, Stange A, Marten I, Bauer H et al (2009) Activity of guard cell anion channel SLAC1 is controlled by drought-stress signaling kinase-phosphatase pair. Proc Natl Acad Sci U S A 106(50):21425-21430. https://doi.org/10.1073/pnas.0912021106

Han S-Y, Shin D, Moon S-J, Jeon S-A, Byun M-O, Kim B-G (2012) Optimization of Agrobacterium-mediated transformation in japonica-type Rice Oryza sativa L. CV. Dongjin for high efficiency. Korean J Breed Sci 44(3):221-228

Hauser F, Waadt R, Schroeder II (2011) Evolution of abscisic acid synthesis and signaling mechanisms. Curr Biol 21(9):R346-R355. https://doi.org/10.1016/j. cub.2011.03.015

Hedrich R, Geiger D (2017) Biology of SLAC1-type anion channels - from nutrient uptake to stomatal closure. New Phytol 216(1):46-61. https://doi. org/10.1111/nph.14685

Hetherington AM, Woodward FI (2003) The role of stomata in sensing and driving environmental change. Nature 424(6951):901-908. https://doi.org/10. 1038/nature01843

Hwang H, Yoon J, Kim HY, Min MK, Kim JA, Choi EH et al (2013) Unique features of two potassium channels, OsKAT2 and OsKAT3, expressed in rice guard cells. PLoS One 8(8):e72541. https://doi.org/10.1371/journal.pone.0072541

Jiao Y, Tausta SL, Gandotra N, Sun N, Liu T, Clay NK et al (2009) A transcriptome atlas of rice cell types uncovers cellular, functional and developmental hierarchies. Nat Genet 41(2):258-263. https://doi.org/10.1038/ng.282

Karimi M, Inze D, Depicker A (2002) GATEWAY vectors for agrobacteriummediated plant transformation. Trends Plant Sci 7(5):193-195

Kim H, Hwang H, Hong JW, Lee YN, Ahn IP, Yoon IS et al (2012) A rice orthologue of the ABA receptor, OsPYL/RCAR5, is a positive regulator of the ABA signal transduction pathway in seed germination and early seedling growth. J Exp Bot 63(2):1013-1024. https://doi.org/10.1093/jxb/err338

Kim HY, Choi EH, Min MK, Hwang H, Moon SJ, Yoon I et al (2015) Differential gene expression of two outward-rectifying shaker-like potassium channels OsSKOR and OsGORK in Rice. J Plant Biol 58(4):230-235. https://doi.org/10. 1007/s12374-015-0070-4

Kim N, Moon SJ, Min MK, Choi EH, Kim JA, Koh EY et al (2015) Functional characterization and reconstitution of ABA signaling components using transient gene expression in rice protoplasts. Front Plant Sci 6:614. https:// doi.org/10.3389/fpls.2015.00614

Kim TH, Bohmer M, Hu H, Nishimura N, Schroeder Jl (2010) Guard cell signal transduction network: advances in understanding abscisic acid, CO2, and Ca2 + signaling. Annu Rev Plant Biol 61:561-591. https://doi.org/10.1146/annurevarplant-042809-112226

Kobayashi Y, Yamamoto S, Minami H, Kagaya Y, Hattori T (2004) Differential activation of the rice sucrose nonfermenting1-related protein kinase2 family by hyperosmotic stress and abscisic acid. Plant Cell 16(5):1163-1177. https:// doi.org/10.1105/tpc.019943

Kusumi K, Hirotsuka S, Kumamaru T, Iba K (2012) Increased leaf photosynthesis caused by elevated stomatal conductance in a rice mutant deficient in SLAC1, a guard cell anion channel protein. J Exp Bot 63(15):5635-5644. https://doi.org/10.1093/jxb/ers216

Lee SC, Lan W, Buchanan BB, Luan S (2009) A protein kinase-phosphatase pair interacts with an ion channel to regulate $A B A$ signaling in plant guard cells. Proc Natl Acad Sci U S A 106(50):21419-21424. https://doi. org/10.1073/pnas.0910601106

Maierhofer T, Diekmann M, Offenborn JN, Lind C, Bauer H, Hashimoto K et al (2014) Site- and kinase-specific phosphorylation-mediated activation of SLAC1, a guard cell anion channel stimulated by abscisic acid. Sci Signal 7(342):ra86. https://doi.org/10.1126/scisignal.2005703

Moon SJ, Kim HY, Hwang H, Kim JA, Lee Y, Min MK et al (2017) A dominant negative OsKAT2 mutant delays light-induced stomatal opening and improves drought tolerance without yield penalty in Rice. Front Plant Sci 8: 772. https://doi.org/10.3389/fpls.2017.00772

Mustilli AC, Merlot S, Vavasseur A, Fenzi F, Giraudat J (2002) Arabidopsis OST1 protein kinase mediates the regulation of stomatal aperture by abscisic acid and acts upstream of reactive oxygen species production. Plant Cell 14(12):3089-3099

Negi J, Matsuda O, Nagasawa T, Oba Y, Takahashi H, Kawai-Yamada M et al (2008) CO2 regulator SLAC1 and its homologues are essential for anion homeostasis in plant cells. Nature 452(7186):483-486. https://doi.org/10.1038/nature06720
Raissig MT, Matos JL, Anleu Gil MX, Kornfeld A, Bettadapur A, Abrash E et al (2017) Mobile MUTE specifies subsidiary cells to build physiologically improved grass stomata. Science 355(6330):1215-1218. https://doi.org/10. 1126/science.aal3254

Saji S, Bathula S, Kubo A, Tamaoki M, Kanna M, Aono M et al (2008) Disruption of a gene encoding C4-dicarboxylate transporter-like protein increases ozone sensitivity through deregulation of the stomatal response in Arabidopsis thaliana. Plant Cell Physiol 49(1):2-10. https://doi.org/10.1093/pcp/pcm174

Schachtman DP, Schroeder JI, Lucas WJ, Anderson JA, Gaber RF (1992) Expression of an inward-rectifying potassium channel by the Arabidopsis KAT1 CDNA. Science 258(5088):1654-1658

Schafer N, Maierhofer T, Herrmann J, Jorgensen ME, Lind C, von Meyer K et al (2018) A tandem amino acid residue motif in guard cell SLAC1 Anion Channel of grasses allows for the control of stomatal aperture by nitrate. Curr Biol 28(9):1370-1379 e1375. https://doi.org/10.1016/j.cub.2018.03.027

Schroeder JI, Allen GJ, Hugouvieux V, Kwak JM, Waner D (2001) Guard cell signal transduction. Annu Rev Plant Physiol Plant Mol Biol 52:627-658. https://doi. org/10.1146/annurev.arplant.52.1.627

Sirichandra C, Wasilewska A, Vlad F, Valon C, Leung J (2009) The guard cell as a single-cell model towards understanding drought tolerance and abscisic acid action. J Exp Bot 60(5):1439-1463. https://doi.org/10.1093/jxb/ern340

Sun SJ, Qi GN, Gao QF, Wang HQ, Yao FY, Hussain J, Wang YF (2016) Protein kinase OsSAPK8 functions as an essential activator of S-type anion channel OsSLAC1, which is nitrate-selective in rice. Planta 243(2):489-500. https://doi. org/10.1007/s00425-015-2418-x

Toki S, Hara N, Ono K, Onodera H, Tagiri A, Oka S, Tanaka H (2006) Early infection of scutellum tissue with agrobacterium allows high-speed transformation of rice. Plant J 47(6):969-976. https://doi.org/10.1111/j.1365-313X.2006.02836.X

Umezawa T, Sugiyama N, Mizoguchi M, Hayashi S, Myouga F, YamaguchiShinozaki K et al (2009) Type 2C protein phosphatases directly regulate abscisic acid-activated protein kinases in Arabidopsis. Proc Natl Acad Sci U S A 106(41):17588-17593. https://doi.org/10.1073/pnas.0907095106

Vahisalu T, Kollist H, Wang YF, Nishimura N, Chan WY, Valerio G et al (2008) SLAC1 is required for plant guard cell S-type anion channel function in stomatal signalling. Nature 452(7186):487-491. https://doi. org/10.1038/nature06608

Vahisalu T, Puzorjova I, Brosche M, Valk E, Lepiku M, Moldau H et al (2010) Ozone-triggered rapid stomatal response involves the production of reactive oxygen species, and is controlled by SLAC1 and OST1. Plant J 62(3):442-453. https://doi.org/10.1111/j.1365-313X.2010.04159.x

Zhang T, Chen S, Harmon AC (2014) Protein phosphorylation in stomatal movement. Plant Signal Behav 9(11):e972845. https://doi.org/10.4161/ 15592316.2014.972845

\section{Submit your manuscript to a SpringerOpen ${ }^{\circ}$ journal and benefit from:}

- Convenient online submission

- Rigorous peer review

- Open access: articles freely available online

- High visibility within the field

- Retaining the copyright to your article

Submit your next manuscript at $>$ springeropen.com 